\title{
Euler Equations on Finite Dimensional Lie Algebras Arising in Physical Problems
}

\author{
O. I. Bogoyavlensky \\ V. A. Steklov Mathematical Institute, Academy of Sciences of the USSR, \\ SU-117966 Moscow, USSR
}

\begin{abstract}
Real physical problems are presented in which Euler equations on Lie algebras of arbitrarily high finite dimension arise. A new integrable case of rotation of a magnetized rigid body in constant gravitational and magnetic fields is found. It generalizes the Kowalewski classical integrable case.
\end{abstract}

\section{Physical Problems Related to Euler Equations on Finite-Dimensional Lie Algebras}

The classical equations describing rotations of a free rigid body around its center of mass were derived by Euler in 1758 [1],

$$
\dot{\mathbf{M}}=\mathbf{M} \times \boldsymbol{\omega},
$$

where $\mathbf{M}$ and $\boldsymbol{\omega}$ are the angular momentum and angular velocity vectors; the equality relating their coordinates is $M_{i}=\sum_{k=1}^{3} I_{i k} \omega_{k}$, where $I_{i k}$ are components of the inertia tensor of the rigid body. From the modern point of view, Eqs. (1.1) are given in the space conjugate to the Lie algebra $\mathrm{SO}(3)$; they are Hamiltonian equations on the orbits $M_{1}^{2}+M_{2}^{2}+M_{3}^{2}=$ const. Arnold [3] proposed the following extension of Euler equations (1.1) for arbitrary Lie algebras,

$$
\dot{\mathbf{M}}=\operatorname{ad}_{a(\mathbf{M})}^{*} \mathbf{M},
$$

where the vector $\mathbf{M}$ belongs to a space $L^{*}$ conjugate to the Lie algebra $L$, and $a(\mathbf{M})$ is a linear self-conjugate (with respect to the natural pairing) operator from $L^{*}$ to $L$. Equations (1.2) are Hamiltonian equations on orbits $\mathcal{O}$ of the co-adjoint representation (in the space $L^{*}$ ) of the Lie group $L$, associated with the Lie algebra $L$. The Hamiltonian for Eq. (1.2), $H=\frac{1}{2}(a(\mathbf{M}), \mathbf{M})$, is necessarily a homogeneous second-order polynomial of components of the vector M. Until recently, there was an opinion that Euler equations in finite-dimensional Lie algebras which can be 
relevant to physical problems must have low dimension, since the physical space is three-dimensional. It has been shown [4] that the classical Kirchhoff equations describing motions of a rigid body in a fluid are Euler equations in the space conjugate to the six-dimensional Lie algebra $E_{3}$ (the Lie algebra for the Lie group $G_{3}$ containing all Euclidean motions of the three-dimensional space $R^{3}$ ). Another known example of Euler-type equations are the equations describing rotation of a rigid body with an ellipsoidal cavity filled with an ideal incompressible fluid which is in homogeneous vortex motion. These equations were introduced by Lamb, Zhukowski, and Poincaré; they are related to the six-dimensional Lie algebra $\mathrm{SO}(3)+\mathrm{SO}(3)=\mathrm{SO}(4)$.

In this section we present new examples of Euler equations on higherdimensional Lie algebras, which arise in real physical problems. An investigation of these examples has revealed that the mentioned definition [3], (1.2), does not include a number of important physical equations related to the Lie algebras. For example, the classical equations describing rotation of a rigid body in a general potential force field, which were also derived by Euler in [1], cannot be represented in the form of (1.2). Therefore in the following we use another, more ample definition of Euler-type equations.

Let $L$ be an $n$-dimensional Lie algebra, $L^{*}$ its conjugate space, $x_{1}, \ldots, x_{n}$ coordinates in $L^{*}$. By definition, the linear functions $x_{1}, \ldots, x_{n}$ belong to the Lie algebra $L$ and form a basis in it; let $C_{i j}^{k}$ be the structure constants of the Lie algebra $L$ in this basis. As is known, a Lie-Poisson bracket exists in the space of functions on $L^{*}$,

$$
\{f, g\}=\sum_{i, j, k=1}^{n} C_{i j}^{k} x_{k} \frac{\partial f}{\partial x_{i}} \frac{\partial g}{\partial x_{j}},
$$

which is related to the Berezin-Kostant-Kirillov symplectic structure in a natural way. Let $H\left(x_{1}, \ldots, x_{n}\right)$ be an arbitrary function in the conjugate space $L^{*}$.

Definition. Euler equations in the space $L^{*}$ are a system of equations written as [12]

$$
d x_{i} / d t=\left\{x_{i}, H\right\}, \quad i=1, \ldots, n .
$$

In particular, if the Hamiltonian $H\left(x_{1}, \ldots, x_{n}\right)$ is a homogeneous second-order polynomial, Eq. (1.4) are reduced to (1.2).

Below we present a list of important physical problems formulated in terms of Euler equations, in the sense of the above definition.

i) Rotation of a rigid body $T$ with a fixed point in a Newtonian field with a potential $\varphi\left(x_{1}, x_{2}, x_{3}\right)$ [5]. Let $\boldsymbol{\alpha}, \boldsymbol{\beta}, \boldsymbol{\gamma}$ be three unit basis vectors of a fixed coordinate frame related to a reference frame $S$ moving with the rigid body. The equations describing rotations of the rigid body in the frame $S$ are

$$
\begin{aligned}
\dot{\mathbf{M}}=\mathbf{M} \times \boldsymbol{\omega}+(\partial U / \partial \boldsymbol{\alpha}) \times \boldsymbol{\alpha}+(\partial U / \partial \boldsymbol{\beta}) \times \boldsymbol{\beta}+(\partial U / \partial \gamma) \times \boldsymbol{\gamma}, \\
\dot{\boldsymbol{\alpha}}=\boldsymbol{\alpha} \times \boldsymbol{\omega}, \quad \dot{\boldsymbol{\beta}}=\boldsymbol{\beta} \times \boldsymbol{\omega}, \quad \dot{\boldsymbol{\gamma}}=\boldsymbol{\gamma} \times \boldsymbol{\omega},
\end{aligned}
$$

where $U(\boldsymbol{\alpha}, \boldsymbol{\beta}, \boldsymbol{\gamma})$ is the potential function

$$
U(\boldsymbol{\alpha}, \boldsymbol{\beta}, \boldsymbol{\gamma})=\int_{T} \varrho(r) \varphi((r, \boldsymbol{\alpha}),(r, \boldsymbol{\beta}),(r, \boldsymbol{\gamma})) d r_{1} d r_{2} d r_{3},
$$

and $r_{1}, r_{2}, r_{3}$ are coordinates in the reference frame $S$. 
Equations (1.5) are Euler equations of the type (1.4) in the space $L_{12}^{*}$, the corresponding Hamiltonian is

$$
H=2^{-1}(\mathbf{M}, \boldsymbol{\omega})-U(\boldsymbol{\alpha}, \boldsymbol{\beta}, \boldsymbol{\gamma}), \quad M_{i}=\sum_{k=1}^{3} I_{i k} \omega_{k} .
$$

The relevant Lie algebra $L_{12}$ is a semi-direct sum $\mathrm{SO}(3)+R^{3}+R^{3}+R^{3}$. In this Lie algebra we have a basis $X_{i}, Y_{j}^{\alpha}(i, j, \alpha, \beta=1,2,3)$, and the commutation relations are

$$
\left[X_{i}, X_{j}\right]=\varepsilon_{i j k} X_{k},\left[X_{i}, Y_{j}^{\alpha}\right]=\varepsilon_{i j k} Y_{k}^{\alpha},\left[Y_{i}^{\alpha}, Y_{j}^{\beta}\right]=0 .
$$

Because of the Laplace equation, $\Delta \varphi=0$, the potential function $U$ satisfies three equations,

$$
\partial^{2} U / \partial \alpha_{i}^{2}+\partial^{2} U / \partial \beta_{i}^{2}+\partial^{2} U / \partial \gamma_{i}^{2}=0, \quad i=1,2,3 .
$$

Evidently, the nonlinearity of the function $U(\boldsymbol{\alpha}, \boldsymbol{\beta}, \boldsymbol{\gamma})$ may be arbitrarily complicated, so in general the Euler equations (1.5) cannot be reduced to (1.2), as in the latter equations the Hamiltonian is a quadratic form. In the simplest case where the potential $\varphi$ is a linear function of coordinates, $\varphi=a^{1} x_{1}+a^{2} x_{2}+a^{3} x_{3}$, Eqs. (1.5) are equivalent to the Euler-Poisson equations; they cannot be transformed to (1.2) in this case either.

ii) Dynamics of a rigid body with a distributed electric charge in ideal incompressible fluid in the presence of constant gravitational and electric fields and under the conditions that the buoyancy and gravity forces are equal and the total charge of the body is zero. In the reference frame $S$, fixed to the body and having its origin at the center of mass, the equations of motion are, if the flow around the body is vortex-free,

$$
\begin{aligned}
\dot{\mathbf{M}} & =\mathbf{M} \times \boldsymbol{\omega}+\mathbf{p} \times \mathbf{u}+m g \mathbf{r} \times \boldsymbol{\gamma}+E \mathbf{d} \times \boldsymbol{\delta}, \\
\dot{\mathbf{p}} & =\mathbf{p} \times \boldsymbol{\omega}, \quad \dot{\boldsymbol{\gamma}}=\boldsymbol{\gamma} \times \boldsymbol{\omega}, \quad \dot{\boldsymbol{\delta}}=\boldsymbol{\delta} \times \boldsymbol{\omega},
\end{aligned}
$$

where $\boldsymbol{\omega}$ is the angular velocity, $\mathbf{u}$ is the velocity of the rigid body in fluid, $\mathbf{p}$ is the total momentum, $\mathbf{M}$ is the total angular momentum (in the frame $S$ ), $m$ is the mass of the body, $g$ is the free-fall acceleration, $\gamma$ indicates the direction of the gravity force, the vector $\mathbf{r}$ stands for the position of the center of mass of the fluid volume displaced by the body, $E$ is the electric field strength, $\mathbf{d}$ is the electric dipole moment vector, and $\boldsymbol{\delta}$ indicates the direction of the electric field. Equations (1.7) are the Euler equations ir space $L_{12}^{*}$; the Hamiltonian is

$$
\begin{gathered}
H=2^{-1} \sum_{i, j=1}^{3}\left(a_{i j} M_{i} M_{j}+2 c_{i j} M_{i} p_{j}+b_{i j} p_{i} p_{j}\right)-m g(\mathbf{r}, \gamma)-E(\mathbf{d}, \boldsymbol{\delta}), \\
\omega_{i}=\partial H / \partial M_{i}, \quad u_{i}=\partial H / \partial p_{i},
\end{gathered}
$$

where $a_{i j}, b_{i j}, c_{i j}$ are arbitrary constant coefficients providing positiveness of the quadratic form. If $E=0,(1.7)$ are the Euler equations in space $L_{9}^{*}$ dual to the Lie algebra $L_{9}$ given by the commutation relations (1.6) for $\alpha, \beta=1,2$. For $E=0, g=0$ equations (1.7) are reduced to the Kirchhoff equations which are Euler equations in $E_{3}^{*}[4]$. 
iii) Rotation of a magnetized rigid body with a fixed point in homogeneous magnetic and gravitational fields is described with the following equations:

$$
\dot{\mathbf{M}}=\mathbf{M} \times \boldsymbol{\omega}+m g \mathbf{r} \times \boldsymbol{\gamma}+h \mathfrak{M} \times \boldsymbol{\delta}, \quad \dot{\gamma}=\boldsymbol{\gamma} \times \boldsymbol{\omega}, \quad \dot{\boldsymbol{\delta}}=\boldsymbol{\delta} \times \boldsymbol{\omega},
$$

where $\mathfrak{M}$ is the magnetic moment vector of the rigid body, the vector $\boldsymbol{\delta}$ indicates direction of the magnetic field, and $h$ stands for the magnetic field strength. Equations (1.8) are the Euler equations in the space $L_{9}^{*}$; the Hamiltonian is

$$
H=2^{-1}(\mathbf{M}, \boldsymbol{\omega})-m g(\mathbf{r}, \gamma)-h(\mathfrak{M i}, \boldsymbol{\delta}), \quad M_{i}=\sum_{k=1}^{3} I_{i k} \omega_{k} .
$$

If $\mathfrak{M}=0$, Eqs. (1.8) are reduced to the classical Euler-Poisson equations which are the Euler equations in $E_{3}^{*}$, in the sense of (1.4).

iv) Dynamics of a rigid body with an ellipsoidal cavity filled with a magnetic fluid in a homogeneous vortex motion (a model of a pulsar rotation) [6]. In the rotating frame $S$ the dynamical equations are

$$
\dot{\mathbf{M}}=\mathbf{M} \times \mathbf{A}, \quad \dot{\mathbf{K}}=\mathbf{K} \times \mathbf{B}+\mathbf{u} \times \mathbf{w}, \quad \dot{\mathbf{u}}=\mathbf{u} \times \mathbf{B},
$$

where $\mathbf{M}$ is the total angular momentum of the rigid body and fluid (referred to their common center of mass), $\mathbf{A}$ is the angular rotation velocity of the rigid body, $\mathbf{B}$ is the angular internal rotation velocity of the fluid, $\mathbf{K}$ stands for the fluid velocity vortex vector, the vectors $\mathbf{u}$ and $\mathbf{w}$ are related to the magnetic field frozen into the fluid. Coordinates of the vectors $\mathbf{A}$ and $\mathbf{B}$ are linear combinations of coordinates of $\mathbf{M}$ and $\mathbf{K}$, respectively; there are also linear relations between coordinates of $\mathbf{u}$ and w [6]. Equations (1.9) are the Euler equations in the space conjugate to the Lie algebra $\mathrm{SO}(3)+E_{3}$; the Hamiltonian is

$$
H=\frac{1}{2}[(\mathbf{M}, \mathbf{A})+(\mathbf{K}, \mathbf{B})+(\mathbf{u}, \mathbf{w})] .
$$

v) Dynamics of a rigid body with $n$ ellipsoidal cavities filled with a magnetic fluid which is in a homogeneous vortex motion is described with the following equations:

$$
\dot{\mathbf{M}}=\mathbf{M} \times \mathbf{A}, \quad \dot{\mathbf{K}}_{\alpha}=\mathbf{K}_{\alpha} \times \mathbf{B}_{\alpha}+\mathbf{u}_{\alpha} \times \mathbf{w}_{\alpha}, \quad \dot{\mathbf{u}}_{\alpha}=\mathbf{u}_{\alpha} \times \mathbf{B}_{\alpha},
$$

where $\alpha=1, \ldots, n, \mathbf{M}$ is the total angular momentum of the body and fluids in all the cavities (referred to the system center of mass), $\mathbf{A}$ is the angular rotation velocity of the rigid body, the vectors $\mathbf{K}_{\alpha}, \mathbf{B}_{\alpha}, \mathbf{u}_{\alpha}, \mathbf{w}_{\alpha}$ are characteristics of the fluid motions and of the frozen magnetic field in the $\alpha^{\text {th }}$ cavity. Coordinates of the vectors $\mathbf{A}, \mathbf{B}_{1}, \ldots, \mathbf{B}_{n}$ are linear combinations of coordinates of the vectors $\mathbf{M}, \mathbf{K}_{1}, \ldots, \mathbf{K}_{n}$, respectively; coordinates of $\mathbf{u}_{\alpha}$ and $\mathbf{w}_{\alpha}$ are also related linearly. Coefficients in the linear relations depend on components of the body inertia tensor and parameters of the ellipsoidal cavities [8]. The notation we use for the relevant Lie algebra is $A_{k, m}=\mathrm{SO}(3)+\ldots+\mathrm{SO}(3)+E_{3}+\ldots+E_{3}$. It is a direct sum of $k$ copies of the SO(3) Lie algebra, and $m$ copies of the $E_{3}$ Lie algebra. Equations (1.10) are the Euler equations in the conjugate space of the Lie algebra $L=A_{1, n}$; the Hamiltonian is

$$
H=\frac{1}{2}\left[(\mathbf{M}, \mathbf{A})+\sum_{\alpha=1}^{n}\left(\left(\mathbf{K}_{\alpha}, \mathbf{B}_{\alpha}\right)+\left(\mathbf{u}_{\alpha}, \mathbf{w}_{\alpha}\right)\right)\right] .
$$


In the absence of magnetic field in $k$ cavities $\left(u_{\alpha}=w_{\alpha}=0\right.$ for $\left.\alpha=1, \ldots, k\right)$ the Lie algebra is reduced to $L=A_{k+1, n-k}$ (the case $k=n$ was considered in [7]).

vi) Rotation of a rigid body with $n$ ellipsoidal cavities, filled with a magnetic fluid, around a fixed point, in a Newtonian field with potential $\varphi\left(x_{1}, x_{2}, x_{3}\right)$, is described with the following equations:

$$
\begin{gathered}
\dot{\mathbf{M}}=\mathbf{M} \times \mathbf{A}+(\partial U / \partial \boldsymbol{\alpha}) \times \boldsymbol{\alpha}+(\partial U / \partial \boldsymbol{\beta}) \times \boldsymbol{\beta}+(\partial U / \partial \gamma) \times \boldsymbol{\gamma}, \\
\dot{\boldsymbol{\alpha}}=\boldsymbol{\alpha} \times \mathbf{A}, \quad \dot{\boldsymbol{\beta}}=\boldsymbol{\beta} \times \mathbf{A}, \quad \dot{\boldsymbol{\gamma}}=\boldsymbol{\gamma} \times \mathbf{A}, \\
\dot{\mathbf{K}}_{\alpha}=\mathbf{K}_{\alpha} \times \mathbf{B}_{\alpha}+\mathbf{u}_{\alpha} \times \mathbf{w}_{\alpha}, \quad \dot{\mathbf{u}}_{\alpha}=\mathbf{u}_{\alpha} \times \mathbf{B}_{\alpha},
\end{gathered}
$$

which are an irreducible combination of Eqs. (1.5) and (1.10). Equations (1.11) are the Euler equations in the space conjugate to the Lie algebra $L=L_{12}+A_{0, n}$; the Hamiltonian is

$$
H=\frac{1}{2}(\mathbf{M}, \mathbf{A})+\frac{1}{2} \sum_{\alpha=1}^{n}\left(\left(\mathbf{K}_{\alpha}, \mathbf{B}_{\alpha}\right)+\left(\mathbf{u}_{\alpha}, \mathbf{w}_{\alpha}\right)\right)-U(\boldsymbol{\alpha}, \boldsymbol{\beta}, \boldsymbol{\gamma}) .
$$

In the absence of magnetic field in $k$ cavities the Lie algebra is reduced to $L=L_{12}$ $+A_{k, n-k}$.

A combination of problems ii) and v) leads to the Euler equations related to the Lie algebra $L=L_{12}+A_{k, n-k}$. In the absence of electric field the Lie algebra $L$ is reduced to $L_{9}+A_{k, n-k}$; if the gravitational field is also vanishing the Lie algebra $L$ is reduced to $E_{3}+A_{k, n-k}=A_{k, n-k+1}$. The Lie algebras $L_{9}+A_{k, n-k}$ and $A_{k, n-k+1}$ are also related to equations arising from combinations of the problems iii) and $v$ ).

\section{A New Integrable Case; Rotation of a Magnetized Rigid Body in Constant Gravitational and Magnetic Fields}

1. Let us consider rotation of a rigid body $T$, having a constant magnetic moment M, with a fixed point 0 , in homogeneous gravitational and magnetic fields. Suppose that in a rotating reference frame $S$ the inertia tensor of the rigid body is diagonal and has components $I_{1}, I_{2}, I_{3}$. To indicate directions of the constant gravitational and magnetic fields we use unit vectors $\gamma$ and $\delta$; the vector $\mathbf{r}$ stands for the position of the center of mass (in the frame $S$ ), $m$ is the rigid body mass, $g$ and $h$ are the gravitational and magnetic field strengths, $\mathbf{M}$ is the angular momentum and $\omega$ is the angular velocity; their components are related by $M_{k}=I_{k} \omega_{k}$. In the frame $S$ the equations of motion have the form of (1.8). The same form is specific also for the equations describing rotation around a fixed point for a charged rigid body with a total charge $\sigma$ in the presence of constant gravitational and electric fields. In the latter case $h$ in Eqs. (1.8) should be replaced by $E$ (the electric field strength), and the vector $\mathfrak{M}$ should be replaced by the electric dipole moment $\mathbf{d}=\int_{T} \sigma(\mathbf{r}) \mathbf{r} d r_{1} d r_{2} d r_{3}$, where $\sigma(\mathbf{r})$ is the electric charge density.

Equations (1.8) have the following first integrals:

$$
J_{1}=2^{-1}(\mathbf{M}, \boldsymbol{\omega})-m g(\mathbf{r}, \boldsymbol{\gamma})-h(\mathfrak{M}, \boldsymbol{\delta}), \quad J_{2}=(\gamma, \gamma), \quad J_{3}=(\boldsymbol{\delta}, \boldsymbol{\delta}), \quad J_{4}=(\boldsymbol{\gamma}, \boldsymbol{\delta}) .
$$


The first integral $J_{1}$ is the total energy of the rigid body. A manifold $\mathscr{M}^{6}$, which is determined by the conditions $J_{2}=1, J_{3}=1, J_{4}=c_{4}$ is in general a product, $\mathscr{M}^{6}=R^{3} \times \mathrm{SO}(3)=T(\mathrm{SO}(3))$ [the tangential bundle to the Lie group $\mathrm{SO}(3)$ ].

As it was mentioned in Sect. 1, Eqs. (1.8) are the Euler equations in the space $L_{9}^{*}$ which is conjugate to the Lie algebra $L_{9}$; the commutation relations for the latter are written in the basis $X_{i}, Y_{j}^{\alpha}$, Eqs. (1.6), where $\alpha, \beta=1,2$. The three dimensional vectors $\mathbf{M}, \boldsymbol{\gamma}, \boldsymbol{\delta}$ belong to subspaces with the basis vectors $X_{i}^{*}, Y_{i}^{1 *}, Y_{i}^{2 *}$, respectively.

For functions in space $L_{9}^{*}$ the Poisson brackets are defined by Eqs. (1.3). For the basis functions we have

$$
\begin{gathered}
\left\{M_{i}, M_{j}\right\}=\varepsilon_{i j k} M_{k}, \quad\left\{M_{i}, \gamma_{j}\right\}=\varepsilon_{i j k} \gamma_{k}, \quad\left\{M_{i}, \delta_{j}\right\}=\varepsilon_{i j k} \delta_{k}, \\
\left\{\gamma_{i}, \gamma_{j}\right\}=\left\{\delta_{i}, \delta_{j}\right\}=\left\{\gamma_{i}, \delta_{j}\right\}=0 .
\end{gathered}
$$

In view of (1.3), the Poisson bracket for arbitrary polynomials of $M_{i}, \gamma_{j}, \delta_{k}$ is calculated using (2.2) by means of the Leibniz rule. The functions $J_{2}, J_{3}, J_{4}$, given in (2.1), annul the Poisson brackets (2.2); manifolds of their combined levels, $\mathscr{M}^{6}$, have a nondegenerate symplectic structure. The equations of motion, (1.8), have a Hamiltonian form,

$$
\dot{M}_{i}=\left\{M_{i}, H\right\}, \quad \dot{\gamma}_{j}=\left\{\gamma_{j}, H\right\}, \quad \dot{\delta}_{k}=\left\{\delta_{k}, H\right\}
$$

with the Hamiltonian function $H=J_{1}$.

Theorem 1. Under the following conditions

$$
m g \mathbf{r}=(R, 0,0), \quad h \mathfrak{M} \mathbf{i}=(0, Q, 0), \quad I_{1}=I_{2}=2 I_{3},
$$

Eqs. (1.8) have also the first integral,

$$
\begin{gathered}
J_{5}=z_{1}^{2}+z_{2}^{2}, \\
z_{1}=M_{1}^{2}-M_{2}^{2}+4 I_{3}\left(R \gamma_{1}-Q \delta_{2}\right), \quad z_{2}=2 M_{1} M_{2}+4 I_{3}\left(R \gamma_{2}+Q \delta_{1}\right) .
\end{gathered}
$$

In an invariant submanifold determined by the condition $J_{5}=0\left(z_{1}=0, z_{2}=0\right)$ there is an additional integral $J_{6}=\left\{z_{1}, z_{2}\right\}$, and Eqs. (1.8) are integrable in Liouville's sense.

In an invariant submanifold determined by the conditions $z_{1}=0, z_{2}=0, J_{6}=0$, Eqs. (1.8) have another additional integral $J_{7}=\left(\left\{J_{6}, z_{1}\right\}\right)^{2}+\left(\left\{J_{6}, z_{2}\right\}\right)^{2}$. In the latter manifold all trajectories of the system (1.8) are closed.

Under the conditions (2.4), Eqs. (1.8) are written as

$$
\begin{aligned}
& \dot{M}_{1}=M_{2} M_{3} / 2 I_{3}+Q \delta_{3}, \quad \dot{M}_{2}=-M_{1} M_{3} / 2 I_{3}-R \gamma_{3}, \quad \dot{M}_{3}=R \gamma_{2}-Q \delta_{1}, \\
& \dot{\gamma}_{1}=\gamma_{2} M_{3} / I_{3}-\gamma_{3} M_{2} / 2 I_{3}, \quad \dot{\gamma}_{2}=\gamma_{3} M_{1} / 2 I_{3}-\gamma_{1} M_{3} / I_{3}, \\
& \dot{\gamma}_{3}=\gamma_{1} M_{2} / 2 I_{3}-\gamma_{2} M_{1} / 2 I_{3}, \quad \dot{\delta}_{1}=\delta_{2} M_{3} / I_{3}-\delta_{3} M_{2} / 2 I_{3}, \\
& \dot{\delta}_{2}=\delta_{3} M_{1} / 2 I_{3}-\delta_{1} M_{3} / I_{3}, \quad \dot{\delta}_{3}=\delta_{1} M_{2} / 2 I_{3}-\delta_{2} M_{1} / 2 I_{3} .
\end{aligned}
$$


The following equations are derived from (2.6)

$$
\begin{aligned}
& \left(M_{1}+i M_{2}\right)=-i\left(2 I_{3}\right)^{-1} M_{3}\left(M_{1}+i M_{2}\right)-i R \gamma_{3}+Q \delta_{3}, \\
& \left(\gamma_{1}+i \gamma_{2}\right)=-i I_{3}^{-1} M_{3}\left(\gamma_{1}+i \gamma_{2}\right)+i \gamma_{3}\left(2 I_{3}\right)^{-1}\left(M_{1}+i M_{2}\right), \\
& \left(\delta_{1}+i \delta_{2}\right)=-i I_{3}^{-1} M_{3}\left(\delta_{1}+i \delta_{2}\right)+i \delta_{3}\left(2 I_{3}\right)^{-1}\left(M_{1}+i M_{2}\right) .
\end{aligned}
$$

Let us multiply the first equation in (2.7) by $2\left(M_{1}+i M_{2}\right)$, the second equation by $4 I_{3} R$, and the third equation by $4 I_{3} Q$. The sum of the resulting equations is

$$
\begin{gathered}
\dot{z}=-i I_{3}^{-1} M_{3} z, \\
z=\left(M_{1}+i M_{2}\right)^{2}+4 I_{3} R\left(\gamma_{1}+i \gamma_{2}\right)+4 i I_{3} Q\left(\delta_{1}+i \delta_{2}\right) .
\end{gathered}
$$

This function can be written as $z=z_{1}+i z_{2}$, where $z_{1}$ and $z_{2}$ are presented in (2.5). The complex equation (2.8) is equivalent to a pair of real equations,

$$
\dot{z}_{1}=\left\{z_{1}, H\right\}=I_{3}^{-1} M_{3} z_{2}, \quad \dot{z}_{2}=\left\{z_{2}, H\right\}=-I_{3}^{-1} M_{3} z_{1} .
$$

A consequence of $(2.8)$ is $(z \bar{z})^{\circ}=\left(z_{1}^{2}+z_{2}^{2}\right)^{\circ}=0$, so $J_{5}=z_{1}^{2}+z_{2}^{2}$ is a first integral for the system (2.6).

Intersections of the manifold $J_{5}=0\left(z_{1}=0, z_{2}=0\right)$ with the submanifolds $\mathscr{M}^{6}$ are four-dimensional symplectic submanifolds $\mathscr{M}^{4}$ (the induced symplectic structure is nondegenerate). In the submanifolds $\mathscr{M}^{4}$ the system (2.6) has an additional first integral,

$$
J_{6}=\left\{z_{1}, z_{2}\right\}=4 M_{3}\left(M_{1}^{2}+M_{2}^{2}\right)+16 I_{3} R M_{1} \gamma_{3}+16 I_{3} Q M_{2} \delta_{3} .
$$

In order to verify this fact one can use Eqs. (2.9) and the Jacobi identity; the result is

$$
\begin{aligned}
\dot{J}_{6} & =\left\{\left\{z_{1}, z_{2}\right\}, H\right\}=-\left\{\left\{z_{2}, H\right\}, z_{1}\right\}+\left\{\left\{z_{1}, H\right\}, z_{2}\right\} \\
& =I_{3}^{-1}\left(z_{1}\left\{M_{3}, z_{1}\right\}+z_{2}\left\{M_{3}, z_{2}\right\}\right)=I_{3}^{-1}\left(2 z_{1} M_{1} M_{2}-z_{2}\left(M_{1}^{2}-M_{2}^{2}\right)\right) .
\end{aligned}
$$

So in submanifolds $\mathscr{M}^{4}$ which are determined by the conditions $z_{1}=z_{2}=0, J_{2}=1$, $J_{3}=1, J_{4}=c_{4}$, one has $\dot{J}_{6}=0$. Thus the Hamiltonian system in the invariant submanifolds $\mathscr{M}^{4}$ has an additional integral $J_{6}$, so it is completely integrable in Liouville's sense.

Let us calculate the time derivatives of functions $\left\{J_{6}, z_{1}\right\}$ and $\left\{J_{6}, z_{2}\right\}$, using the Jacobi identity,

$$
\left\{\left\{J_{6}, z_{i}\right\}, H\right\}+\left\{\left\{z_{i}, H\right\}, J_{6}\right\}+\left\{\left\{H, J_{6}\right\}, z_{i}\right\}=0, \quad i=1,2 .
$$

Putting Eqs. (2.9)-(2.11) into (2.12) we get

$$
\begin{aligned}
\left\{J_{6}, z_{1}\right\}^{\prime}= & \left\{\left\{J_{6}, z_{1}\right\}, H\right\}=I_{3}^{-1}\left(z_{2}\left\{J_{6}, M_{3}\right\}+2 z_{1}\left\{M_{1} M_{2}, z_{1}\right\}\right. \\
& \left.+z_{2}\left\{z_{1}, M_{1}^{2}-M_{2}^{2}\right\}+J_{6}\left(M_{1}^{2}-M_{2}^{2}\right)+M_{3}\left\{J_{6}, z_{2}\right\}\right), \\
\left\{J_{6}, z_{2}\right\}^{\prime}= & \left\{\left\{J_{6}, z_{2}\right\}, H\right\}=I_{3}^{-1}\left(z_{1}\left\{M_{3}, J_{6}\right\}+2 z_{1}\left\{M_{1} M_{2}, z_{2}\right\}\right. \\
& \left.+z_{2}\left\{z_{2}, M_{1}^{2}-M_{2}^{2}\right\}+2 J_{6} M_{1} M_{2}-M_{3}\left\{J_{6}, z_{1}\right\}\right) .
\end{aligned}
$$

In the invariant manifold determined by the conditions $z_{1}=0, z_{2}=0, J_{6}=0$, Eqs. (2.13) acquire the form

$$
\left\{J_{6}, z_{1}\right\}^{\cdot}=I_{3}^{-1} M_{3}\left\{J_{6}, z_{2}\right\}, \quad\left\{J_{6}, z_{2}\right\}^{\cdot}=-I_{3}^{-1} M_{3}\left\{J_{6}, z_{1}\right\} .
$$


Consequently, under the conditions $z_{1}=0, z_{2}=0, J_{6}=0$ the system described by Eqs. (2.6) has the additional integral $J_{7}=\left(\left\{J_{6}, z_{1}\right\}\right)^{2}+\left(\left\{J_{6}, z_{2}\right\}\right)^{2}$. In every invariant four-dimensional submanifold $\mathscr{M}^{4}\left(z_{1}=0, z_{2}=0, J_{2}=1, J_{3}=1, J_{4}=c_{4}\right)$, the intersection of three level surfaces $J_{1}=c_{1}, J_{6}=0, J_{7}=c_{7}$ determines closed trajectories of the system (2.6). This completes the proof of Theorem 1.

In the absence of the magnetic field $(Q=0)$ the integrable case specified by Eq. (2.4) for the system (1.8) is reduced to the classical case discovered by Kowalewski [2]. In the integrable case obtained here the potential function $U=R \gamma_{1}+Q \delta_{2}$ [cf. Eq. (1.5)] has an essential dependence on three Euler angles $\varphi$, $\psi, \theta$. Integrable cases where the function $U$ depends on two Euler angles $\varphi, \theta$ were investigated by Goryachev $[9,10]$.

Remark. It is not difficult to verify by means of a direct differentiation that Eqs. (2.6) result in a matrix Lax equation,

$$
\dot{L}=[L, A] \text {, }
$$

where matrices $L$ and $A$ are two-dimensional and have the following elements $l_{i j}, a_{i j}$,

$$
\begin{gathered}
l_{11}=M_{2}^{2}+M_{3}^{2}-4 I_{3} R \gamma_{1}, \quad l_{22}=M_{1}^{2}+M_{3}^{2}-4 I_{3} Q \delta_{2}, \\
l_{12}=l_{21}=-M_{1} M_{2}-2 I_{3}\left(R \gamma_{2}+Q \delta_{1}\right), \\
a_{11}=a_{22}=0, \quad a_{21}=-a_{12}=\left(2 I_{3}\right)^{-1} M_{3} .
\end{gathered}
$$

Because of Eqs. (2.15), the functions $\operatorname{Tr}(L)$ and $\operatorname{Det}(L)$ are first integrals of the system (2.6). These integrals are related to the integrals $J_{1}$, Eq. (2.1), and $J_{5}$, Eq. (2.5),

$$
J_{1}=\left(4 I_{3}\right)^{-1} \operatorname{Tr}(L), \quad J_{5}=(\operatorname{Tr}(L))^{2}-4 \operatorname{Det}(L) .
$$

If $Q=0$, the matrix equations (2.15)-(2.16) are reduced to an equation derived for the Kowalewski case in [11].

\section{References}

1. Euler, L.: Decouverte d'une nouveau principe de mechanique. Mem. Acad. Sci. Berlin 14, 154-193 (1758)

2. Kowalewski, S.V.: Sur la probleme de la rotation d'un corps solide autour d'un point fixe. Acta Math. 12, 177-232 (1889)

3. Arnold, V.I.: Mathematical methods of classical mechanics (in Russian). Moscow: Nauka 1974 [English transl. Berlin, Heidelberg, New York: Springer 1979]

4. Novikov, S.P., Shmeltser, I.: Periodical solutions of the Kirchhoff equations for free motion of a rigid body in fluids and an extended Lyusternik-Shnirelman-Morse theory. I (in Russian). Funkcional. Anal. i Prilozen. 15, 54-66 (1981)

5. Bogoyavlensky, O.I.: New integrable problem of classical mechanics. Commun. Math. Phys. 94, 255-269 (1984)

6. Bogoyavlensky, O.I.: Periodic solutions in a model of pulsar rotations. Commun. Math.Phys. (submitted)

7. Bogoyavlensky, O.I.: Integrable Euler equations on $\mathrm{SO}(4)$ and their physical applications. Commun. Math. Phys. 93, 417-436 (1984) 
8. Bogoyavlensky, O.I.: Dynamics of a rigid body with $n$ ellipsoidal cavities filled with a magnetic fluid (in Russian). Dokl. Akad. Nauk SSSR 272, 1364-1367 (1983)

9. Goryachev, D.N.: New cases of motion of a rigid body around a fixed point (in Russian). Warsaw University Izv. 3, 3-14 (1915)

10. Goryachev, D.N.: New cases of integrability for dynamical Euler equations (in Russian). Warsaw University Izv. 3, 3-15 (1916)

11. Perelomov, A.M.: Lax representation for the system of S. Kowalevskaya type. Commun. Math. Phys. 81, 239-243 (1981)

12. Berezin, F.A.: Models os Gross-Neveu type are quantization of a classical mechanics with nonlinear phase space. Commun. Math. Phys. 63, 131-154 (1978)

Communicated by Ya. G. Sinai

Received April 25, 1984 
